\title{
"Little Old Ladies Hernia": A Case Report and Review of Literature
}

\author{
${ }^{1}$ Department of Radiodiagnosis, Fortis Hospital, India \\ ${ }^{2}$ Department of gastrointestinal surgery, Apollo hospitals, India \\ ${ }^{3}$ Department of Surgical Gastroenterology, Fortis Hospital, India
}

Sonali Sethi ${ }^{1 *}$, SatyajitGodhi' ${ }^{2}$, Pankaj Kumar ${ }^{1}$ and Amit Javed ${ }^{3}$

*Corresponding author: Sonali Sethi, Consultant Radiologist, Department of Radiodiagnosis, Melody 1002, H M Tambourine Apartments JP Nagar 6th phase, Kanakpura Road, Bangalore, Karnataka. -560078, India

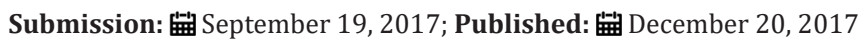

\begin{abstract}
Obturator hernia is a very rare variety of pelvic hernia accounting for approximately $0.05-0.4 \%$ of all abdominal hernias[1].It is referred to as the "little old ladies hernia" owing to its prevalence in old frail ladies. The symptoms are non-specific and the diagnosis is often delayed. We recently managed an 83 year old lady who presented with intestinal obstruction due to an incarcerated left obturator hernia. She was diagnosed preoperatively with a contrast enhanced computed tomography (CECT) abdomen and operated successfully by laparoscopic surgery. The radiologist and clinician should be aware of this clinical entity as it most often presents very late (as in our case) and early diagnosis is imperative for successful management and better prognosis.
\end{abstract}

Case Report

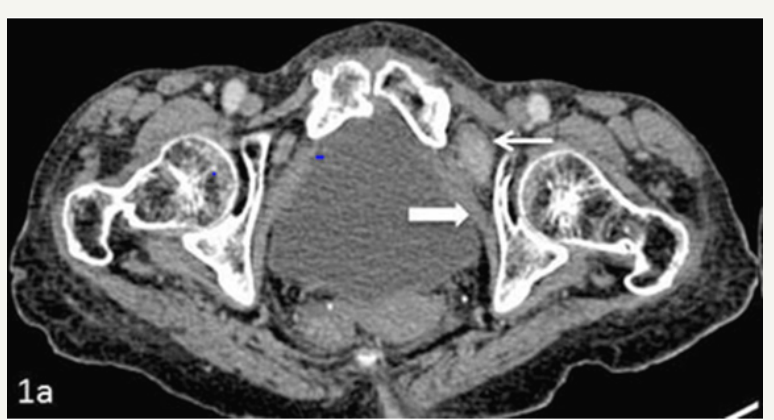

Figure 1a: Axial CECT abdomen is seen in through the lower pelvis showing herniation of mid lleal loop through the left obsturator foramen (thin white arrow). The obturator internus muscle is seen posteriorly (bold white arrow).

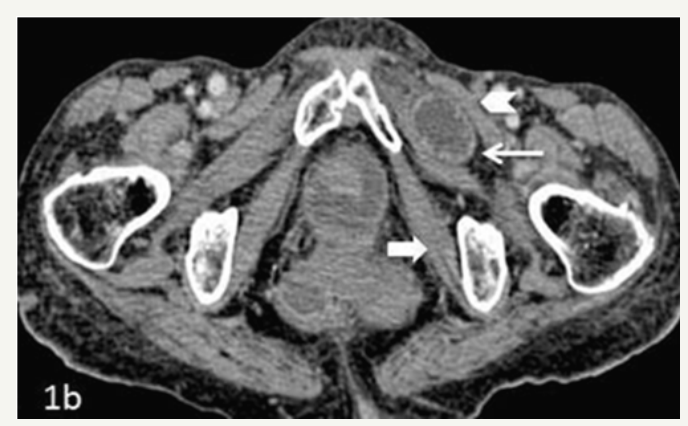

Figure 1b: Axial sections at a caudal level show the incarcerated segment of the herniated loop in the upper thigh anteriorly. The pectineus muscle is seen anterior to the herniated bowel (arrow head) and the obturatorinternus muscle is seen posteriorly (bold white arrow).

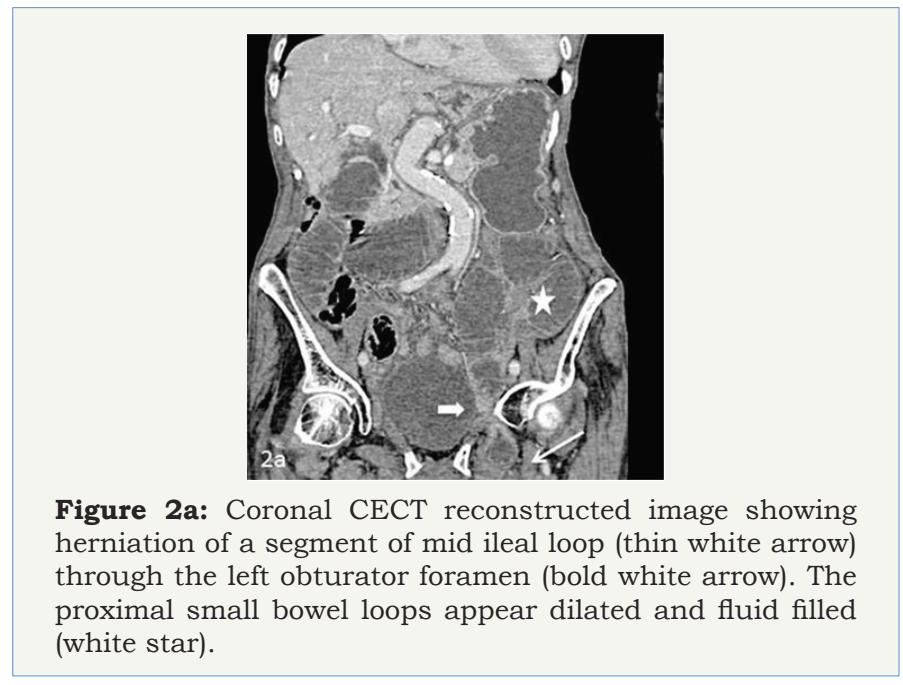

An 83 year old lady presented with complaints of acute pain in abdomen and left anterior thigh with non-passage of stools and flatus for the past two days. She had previous history of ischemic heart disease for which she had undergone coronary stenting and pulmonary tuberculosis for which she had completed antitubercular treatment. There was no past history of any abdominal surgery. On examination, she had a thin built with a BMI of 17 . At presentation she was dehydrated and pale. Her abdomen was distended, mildly tender with exaggerated bowel sounds. The hernial orifices were clear and rectal and vaginal examinations were unremarkable. No mass was felt at the site of pain in the left anterior thigh. She was resuscitated and underwent an abdominal radiograph that showed multiple air fluid levels and dilated small 
bowel loops suggestive of mechanical small bowel obstruction. Subsequently, CECT of abdomen was performed which showed dilatation of the small bowel loops (measuring approximately 3-3.5 $\mathrm{cm}$ in caliber) with multiple internal air fluid levels. There was herniation of a mid ileal loop for a length of approximately $2.3 \mathrm{~cm}$ through the left obturator foramen and was lying in the anterior thigh between the pectineus and obturatorexternus muscles (Figure $1 \& 2$ ). The bowel wallshowed normal CT morphology with no features to suggest infarction.

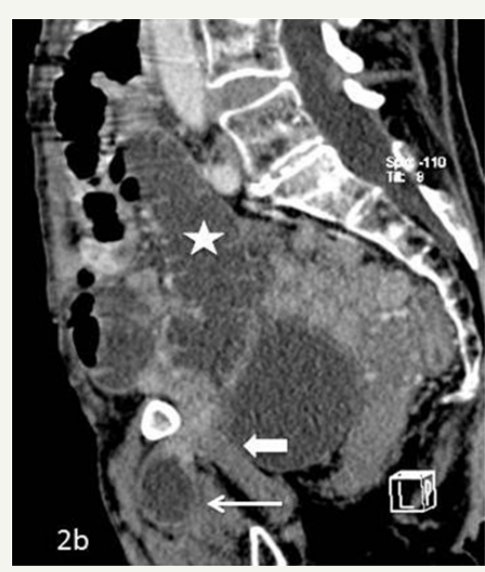

Figure 2b: Sagittal image of CECT abdomen showing herniation of the mid ileal bowel loop (thin white arrow) through the left obturator foramen. The obturatorinternus muscle is seen posteriorly (bold white arrow). The proximal small bowel loops appear dilated and fluid filled (white star).

The patient was taken up for emergency laparoscopic surgery which confirmed the diagnosis of incarcerated left obturator hernia. The entrapped segment of ileum was released (Figure $3 A, B)$. The stuck segment of the bowel was edematous however; there was no evidence of ischemia. The fascial defect was repaired by interrupted sutures (Figure 4). The post-operative course was uneventful and the patient was discharged on the fourth postoperative day. At the end of two years patient is asymptomatic with no hernia recurrence.

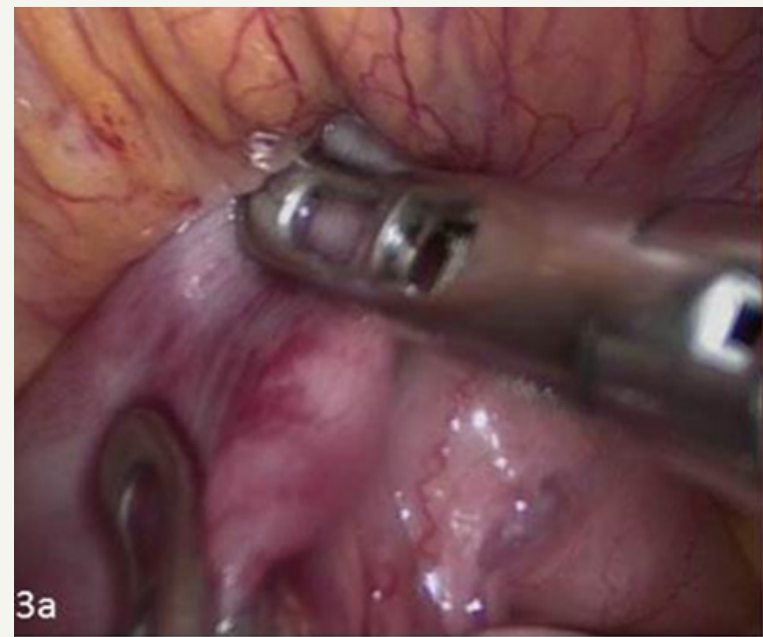

Figure 3a: Intra-operative image showing incarcerated segment of small bowel in the left obturator foramen.

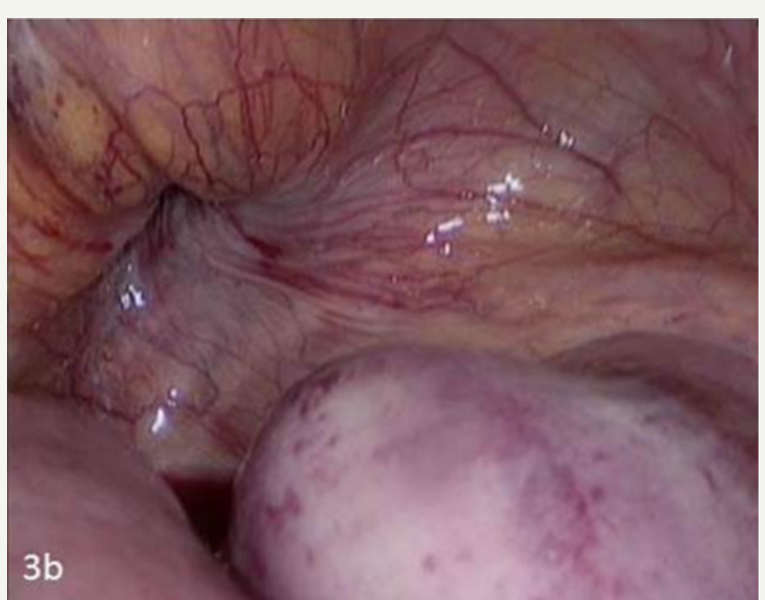

Figure 3b: The fascial defect is seen involving the left obturator foramen.

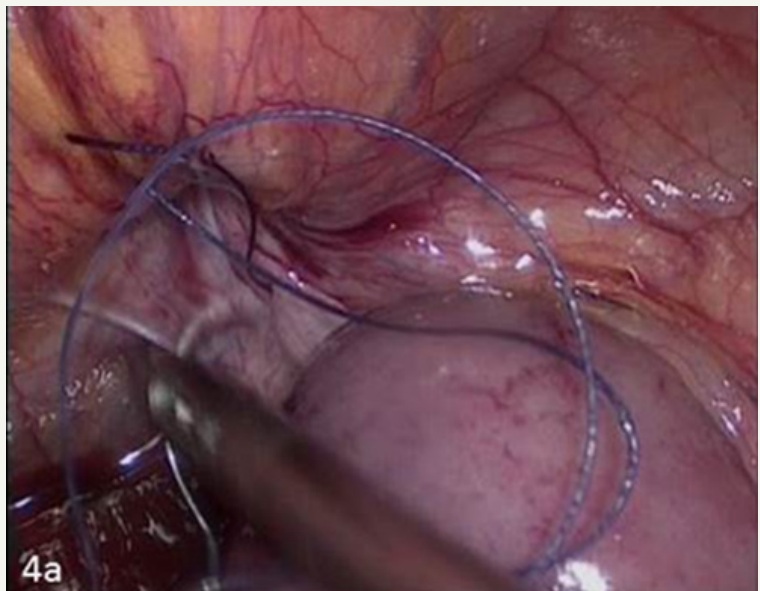

Figure 4a: The facial defect is being closed by interrupted sutures.

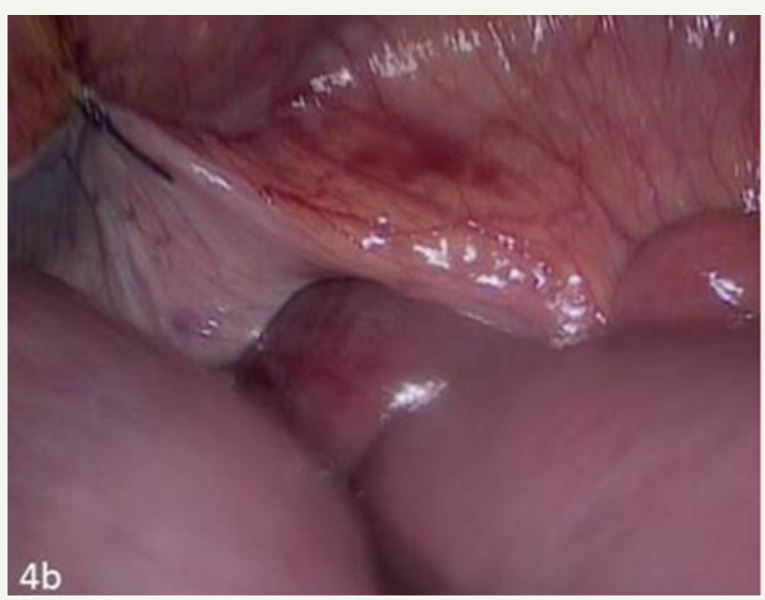

Figure 4b: The facial defect is being closed by interrupted sutures.

\section{Discussion}

Obturator hernia is a rare type pelvic hernia, accounting for $0.05-0.4 \%$ of abdominal hernias [1]. It was first described by 
Armaud de Ronsil and first treated by Henry Obre in the years 1724 and 1851 respectively [2]. It accounts for $0.2-1.6 \%$ cases of mechanical small bowel obstruction [3]. The associated mortality with this hernia is between $13-40 \%$ and is the highest amongst all hernias due to delayed diagnosis owing to non-specific often intermittent symptoms, unusual location and co-morbidities in the elderly. The female to male ratio is 6-9:1 due to wider and more oblique obturator canal in the females [4]. Right sided obturator hernias are more common due to the protective effect of the sigmoid colon on the left [5]. In contrast, the hernia was on the left sided in our patient and may have been secondary to the redundant sigmoid colon. Bilateral hernias can be seen in $6 \%$ of the patients at initial presentation. Coexisting hernias like femoral and inguinal have also been reported [4].

Risk factors include old age, post-part-time status, multi-parity and raised intra-abdominal pressure, rapid weight loss with loss of pre peritoneal fat surrounding the obturator canal.The risk factors in our patient included old age, multi-parity, cough from her prior pulmonary tuberculosis and asthenia.

The symptoms are often paroxysmal like intermittent abdominal pain, nausea and vomiting owing to Richter's herniation in the initial stages. The pain is provoked or worsened by coughing and straining and may be attributed falsely to osteoarthritis which is common in this age group. These symptoms are often ignored. Advanced cases present with features related to strangulation and small bowel obstruction which is the usual presentation in $90 \%$ cases as was in our case as well [4]. Specific signs related to the hernia have been described like radiating pain down the medial aspect of the thigh up to the knee known as the Howship-Romberg sign which is related to the irritation of the anterior division of the obturator nerve. However this obturator neuralgia is not very sensitive and is present in $12.5 \%$ to $65 \%$ cases. Loss of the adductor reflex of the thigh in the presence of a positive patellar reflex called the Hannigton-Kiff sign is more specific and is used to differentiate the pain from that due to osteoarthritis. In cases with high index of suspicion the obturator region should be palpated and it might reveal a palpable mass or elicit tenderness.

The initial investigation is often a plain radiograph of the abdomen that shows dilated bowel loops with air fluid levels in the setting of acute obstruction. It is however, difficult to elucidate the exact etiology of obstruction. The preoperative diagnosis by USG has been described by XueHeng et al using a high frequency transducer which may reveal the incarcerated segment in the upper thigh [6]. Ultrasound is widely available, cost effective and often the first modality used in abdominal symptoms. However, it is operator dependent, is less sensitive for an entity like obturator hernia due to its atypical and deep location. The diagnosis is not possible in early stages by ultrasonography and sensitivity is also reduced if too much pressure is applied on the transducer. A barium enema and meal follow through also has a limited role in acute setting and may delay intervention due to slow flow of barium in the setting of obstruction. The hernia may also be misdiagnosed as femoral or inguinal. There is limited role of MRI and herniography in an acute
setting.Pre-operative diagnosis was uncommon before the advent of CT scanner. CT scan has a very high diagnostic value 78-100\% [7] and has become the gold standard for the pre-operative diagnosis. Its role was first described by Cubillo in 1983 [8]. CT can accurately make the diagnosis, can identify the hernia contents, differentiate other causes of intestinal obstruction and identify signs of bowel ischemia like hyper or hypo enhancement, bowel wall thickening and mesenteric stranding. Signs of perforation like pneumoperitoneum and free fluid can also be accurately identified.

Exploratory laparoscopy is the gold standard for confirming the diagnosis and management by reduction and repair of the hernia. It however, requires expertise which is not available in most peripheral centres. The initial port insertion may be technically more challenging in cases with hugely dilated bowel and open technique is preferable. Compared to open surgery, it is less invasive with less morbidity and fewer post-operative pulmonary complications, ileus, pain and shorter hospital stay duration which is important in the subset of geriatric patients. $\mathrm{Ng}$ et al have reported lesser complications in laparoscopic approach compared to open technique in patients who underwent obturator hernia surgery for over a 15 year period [9].

The abdominal approach with low midline incision is the most popular approach in literature. It allows better exposure of the obturator ring, avoids the obturator vessels, and facilitates bowel resection if required. Retropubic, transperitoneal inguinal and obturator are the other surgical approaches. A high inguinal approach was used by Hodgin's et al. [10] to successfully repair an obturator hernia reported pre-operatively as femoral .

Extra peritoneal approach may be utilized in diagnosed cases with no obstruction. Simple closure of the hernia defect with interrupted sutures and mesh repair are the surgical options. Simple closure has the advantage of faster surgery and hence lesser post-operative morbidity. Acceptable recurrence rate of $10 \%$ has been seen with the simple closure technique. Mesh hernia repair may entail complication like post-operative infection of the mesh in cases requiring bowel resection.

\section{Conclusion}

Obturator hernia is a rare cause of small bowel obstruction in the elderly and entails a significant mortality and morbidity which is highest amongst all abdominal hernias. It is important for the surgeon and radiologist to be abreast with the clinical symptoms, radiological findings and surgical approaches for its rapid diagnosis and treatment.

\section{References}

1. Wu JM, Lin HF, Chen KH, Tseng LM, Huang SH (2006) Laparoscopic preperitoneal mesh repair of incarcerated obturator hernia and contralateral direct inguinal hernia.J Laparoendosc Adv Surg Tech A 6(6): 616-619.

2. Kammori M, Mafune K, Hirashima T, Kawahara, Hashimoto M, et al. (2004) Fourty three cases of obturator hernia. Am J Sur 187(4): 549552. 
3. Khaladkar SM, Kamal A, Garg S, Kamal V (2014) Bilateral obturator hernia diagnosed by CT. Radiol Res Pract 2014: 625873.

4. Markevicious M, Lunevicius R, Markovas V, Stanaitis J (2010) Incarcerated obturator hernia in 49 year year old women: a case report and review of the literature. Lithuanian Surgery 8(4): 260-264.

5. Aydin I, YucelAF, Pergel A, Sahin D (2013) Obturator hernia: a rare case of acute mechanical intestinal obstruction. Case Rep Sur 2013:256062.

6. Heung, XueXue H, Fu Y, Cui LG (2016) Small bowel obstruction secondary to obturator hernia pre-operatively diagnosed by ultrasound. Chin Med J (Engl) $201620^{\text {th }}$ Feb;129(4): 490-491.
7. Kisaoglu A, Ozogul B, Yuce I, Bayramoglu A, Atamanalp SS (2014) Obturatorhernia, a rare cause of small bowel obstruction: case report. Eurasian J Med 46(3): 224-226.

8. Yokoyama y, YamaguchiA, Isogai M, Hori, Kaneoka (1999) Thirty six cases of obturator hernia: does computed tomography contribute to post-operative outcome? World J Surg 23(2): 2144-2147.

9. Ng DC, Tang CN, Li MK. (2014) Fifteen-year experience in managing obturatorhernia : from open to laproscopic approach, 18:381-386.

10. Hodgins N, Cieplucha K, Conneally P, Ghareeb E (2013) Obturator hernia: A Case report and review of literature. Int J Surg Case Rep 4(10) : 889892. 